\title{
Eccentric-concentric Ratio: A Key Factor for Defining Strength Training in Soccer
}

\author{
Authors \\ F. Javier Nuñez ${ }^{1}$, Moisés de Hoyo² ${ }^{2}$ Alejandro Muñoz López ${ }^{3}$, Borja Sañudo², Carlos Otero-Esquina ${ }^{4}$, Hugo Sanchez ${ }^{4}$, \\ Oliver Gonzalo-Skok 5
}

\section{Affiliations}

1 Physical Performance \& Sports Research, University of Pablo de Olavide, Seville, Spain

2 Physical Education and Sport, University of Seville, Seville, Spain

3 Sports and Computing, University Pablo de Olavide, Seville, Spain

4 Fitness Section, Sevilla Football Club, Seville, Spain

5 Faculty of Health Sciences, University of San Jorge, Zaragoza, Spain

\section{Key words}

rotatory inertial device, mean power, sprint performance, force-vector training, resistance training, young soccer players

accepted 15.07.2019

\author{
Bibliography \\ DOI https://doi.org/10.1055/a-0977-5478 \\ Published online: 21.8 .2019 \\ Int J Sports Med 2019; 40: 796-802 \\ (c) Georg Thieme Verlag KG Stuttgart · New York \\ ISSN 0172-4622
}

\section{Correspondence}

Dr. Javier Nuñez

Department of Sports and Informatics

University of Pablo de Olavide of Sevilla

Carretera de Utrera km 1

41013 Sevile

Spain

Tel.: + 34/606/204 313, Fax: + 34/954/977 534

fjnunsan@upo.es

\begin{abstract}
The aims of this study were to analyse the effect of chronic strength training over concentric power (CON), eccentric power (ECC), ECC/CON ratio, and $20 \mathrm{~m}$ linear sprint performance in elite young soccer players. Twenty young elite Spanish soccer players were assigned to an experimental group (CPG) which performed a front-step exercise using a conical pulley, $2-3$ sets of 6 repetitions each leg, during 9 weeks (CPG, $n=10$ ) in addition to its usual strength training, or to a control group (CG, $\mathrm{n}=10)$. The improvements in the ECC mean power $(36 \%$, $E S=1.61)$, and $E C C / C O N$ ratio (17\%, $E S=1.77)$ were substantially greater in the CPG than in the CG while the CON mean power $(16 \%, E S=0.83)$ was substantially greater in the CG than in the CPG. The sprinting time for $10 \mathrm{~m}(2.8 \%, E S=0.78)$ and the $10 \mathrm{~m}$ flying time between $10-20 \mathrm{~m}(1.72 \%$, ES $=0.41$ ) were substantially enhanced in CPG and CG respectively. To be efficient when defining a functional strength training and performance increments using an inertial device, the mean power output need to be measured during the CON and ECC phases and an analysis of the ECC / CON ratio should be included.
\end{abstract}

\section{Introduction}

Sprint running acceleration is a key feature of physical performance in team sports [1]. The main determinant of the sprint-acceleration profile is the ability to produce high levels of mechanical power output [2]. It has been recently shown that power output in this task primarily depends on ground reaction forces in the postero-anterior direction but only if the tasks are performed at high contraction velocities $[1,2]$. Most of the training methods used for sprint improvement (e. g., sled training, squats, and plyometric techniques) involve a high degree of stimulation during the concentric (CON) phase, and it seems that eccentric (ECC) contractions are under-activated $[3,4]$. The rotatory inertial devices have been designed to provide a maximal resistance load during the CON phase, and they offer a substantially higher ECC load than free weight exercises [5]. Training with these devices elicits higher gains in terms of muscle power and sprinting efficiency, when compared with traditional weight-stack exercises [6]. 
Recently, De Hoyo et al. [7] analyzed the effects of an 8-week (2 times per week) training programme, which included moderate sled training, full squats, and plyometric exercise, on sprinting abilities in young soccer players. Only the full squat training group was able to improve their sprint performances (e. g., times between $10-20 \mathrm{~m}$ ), although improvements in 10 and $20 \mathrm{~m}$ sprints were not significant. Otero-Esquina et al. [8] examined the effects of combined sled training, full squats, and plyometric exercises with a leg curl inertial device exercise that has a specific involvement of the hamstring muscles during the ECC phase [9]. These authors showed improvements in $20 \mathrm{~m}$ sprints in young soccer players after 7 weeks of training but only in those subjects who trained 2 times a week and not in those subjects who trained only once a week or the control group; therefore, it could be suggested the incorporation of this inertial device may improve several abilities in young soccer players. Recent studies using other inertial devices (e. g., the "conical pulley") have reported improvements in sprint abilities (from $0-25 \mathrm{~m}$ ) in team sports [10]; however, the results in young soccer players are not conclusive [11].

The rotatory inertial device provides a source of linear resistance from a tether wrapped around a vertical cone-shaped shaft. The tether winds around the cone, and the CON action unwinds the tether, whereas the ECC action occurs during rewinding. The kinetic energy from the CON portion of the exercise is transferred to the ECC portion; an equal ECC impulse is necessary to halt the rotation of the disc [5]. The use of the conical pulley device for training tends to be most efficient when the athlete applies force throughout the course of the full CON phase at maximal velocity [5]. Nevertheless, the athlete can halt the rotation during the ECC phase by: a) increasing the damping time in the eccentric phase so that this load will be attenuated; b) equalizing the time of the CON and ECC phases, which would result in similar concentric and eccentric loads; and c) reducing the time of the ECC phase with respect to that of the CON phase, which can be achieved by an accompanying movement without a slowing of the movement in the first portion, as well as by reducing the energy of the cone only at the end of the action.

Usually, improvements in inertial device performance are evaluated based on the increases in the mean and maximal power values during the CON and ECC phases of the inertial exercise [12]. If the athlete halts the rotation during the ECC phase in a different manner, each manner could influence the ability to generate power in the concentric phase of the next repetition. Thus an analysis of the evolution of the training process, based on the concentric or eccentric power values, without an analysis of how they are related, could generate a loss of valuable information for training controls. Accordingly, the aims of this study were to analyze the effects of inertial chronic strength training on concentric power (CON), eccentric power (ECC), ECC/CON ratio, and $20 \mathrm{~m}$ linear sprint performances in elite young soccer players.

\section{Materials and Methods}

\section{Subjects}

Twenty young, elite, Spanish soccer players (values in mean \pm SD; age: $17 \pm 1$ years, height: $178.1 \pm 2.3 \mathrm{~cm}$, body mass: $62.8 \pm 6.6 \mathrm{~kg}$, and 1-RM full-squat: $105.3 \pm 23.7 \mathrm{~kg}$ ) were recruited to participate in this study. One CON repetition maximum (1-RM) from the full squat exercise was determined according to the guidelines proposed by de Hoyo et al. [13]. The barbell was attached to a dynamic measurement system (T-Force System, Ergotech, Murcia, Spain). The athletes belonged to a first Spanish soccer division (Liga Santander) club academy squad. All of the participants were involved in the typical soccer training regimen, with a similar weekly training volume (4-5 sessions/week of 60-90 min and 1 match per week). The study was conducted according to the Declaration of Helsinki [14], the protocol was fully approved by the local research ethics committee before the recruitment of the players, and meets the ethical standards of the journal [15].

After a detailed explanation of the aims, benefits, and risks involved in this investigation, all of the participants and their parents provided written informed consent.

\section{Procedures}

Each participant visited the gym facility and completed a familiarization session 7 days before the pre-test. During the familiarization session, a full explanation of the experimental protocol was provided to the participants, and they were permitted to practice all of the tests. Players preformed one testing session by performing tests in the following order: a 20 m linear sprint test and a lower-limb power test. Tests were separated by 3 min rest periods. After 9 weeks, a post-test was performed under the same conditions as the pre-test. To compare the effects of 9 weeks of the addition of a weekly conical pulley training regimen to the usual strength training regimen, including moderate sled training, full squats, and plyometric exercises (as described by Otero-Esquina et al. [8]), participants were randomly allocated to: a) the experimental group (CPG, $n=10$ ), which included the addition of the aforementioned strengthening programme with an one-step-acceleration exercise by using the conical pulley device, as described by De Hoyo et al. [13]; and b) a control group (CG, $n=10)$, which only performed the usual strength training sessions during the training period.

After a standardized warm-up period, the CPG group performed a weekly, one-step-acceleration exercise with a conical pulley device, in addition to the usual strength training sessions. This exercise consisted of 2-3 sets of 6 repetitions of each leg at loads that elicited the MP CON phase. The strength training sessions involved an increasing volume programme, as shown in \ Table 1.

\section{0-metre linear sprint test}

Subjects were assessed in a 20 -m linear sprint test, and times were recorded with the use of photoelectric cells (Racetime2, Microgate ${ }^{\circledR}$, Bolzano, Italy) at distances of 0,10 , and $20 \mathrm{~m}$. The preferred front foot was placed $0.5 \mathrm{~m}$ behind the first timing gate, and players started the sprints when they were ready, with an avoidace of the reaction time. Subjects were given two practice trials after a warm-up, to familiarize them. After 3 min of passive rest, four trials were completed, and the best $20 \mathrm{~m}$ performance trial was used for the subsequent statistical analysis. The sprinting time for $10 \mathrm{~m}$ (T10m) and $20 \mathrm{~m}$ (T20m) sprints, as well as the $10 \mathrm{~m}$ flying time (T10-20 $\mathrm{m}$ ) were also considered for further analysis. Two min of passive rest was permitted between the trials. 


\begin{tabular}{|c|c|c|c|c|c|c|c|c|}
\hline $\begin{array}{l}a \\
\stackrel{9}{\sharp} \\
3\end{array}$ & 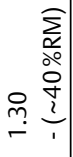 & 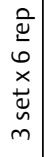 & 仓̊ํ & 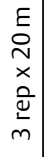 & $\begin{array}{l}z \\
\text { ப } \\
\vdots \\
\Sigma\end{array}$ & 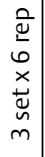 & & \\
\hline 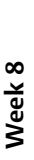 & 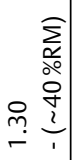 & 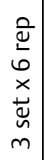 & $\stackrel{\circ}{\stackrel{人}{~}}$ & 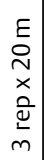 & $\begin{array}{l}z \\
0 \\
\dot{y} \\
\bar{z}\end{array}$ & 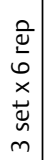 & 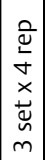 & \\
\hline $\begin{array}{l}\tilde{y} \\
\text { ¿ूँ }\end{array}$ & 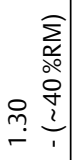 & 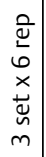 & @̊ & 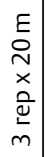 & $\begin{array}{l}z \\
\dot{U} \\
\grave{z}\end{array}$ & 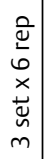 & 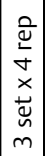 & \\
\hline 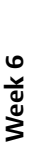 & 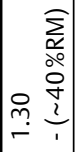 & 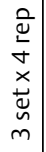 & $\stackrel{\circ}{\stackrel{ }{N}}$ & 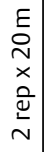 & $\begin{array}{l}z \\
\dot{u} \\
\grave{z}\end{array}$ & $\begin{array}{l}\stackrel{a}{\omega} \\
\dot{b} \\
\times \\
\stackrel{ \pm}{\omega} \\
m\end{array}$ & 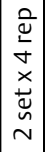 & \\
\hline 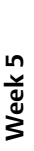 & 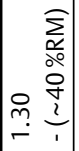 & 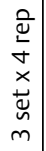 & $\stackrel{\circ}{\stackrel{人}{~}}$ & 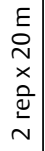 & $\begin{array}{l}z \\
\dot{u} \\
\grave{\Sigma}\end{array}$ & 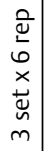 & & \\
\hline 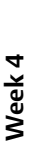 & 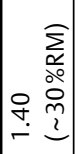 & 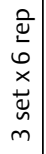 & 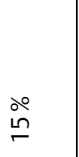 & 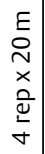 & $\mid \begin{array}{l}z \\
0 \\
0 \\
\vdots \\
z\end{array}$ & 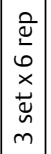 & 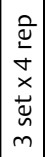 & \\
\hline 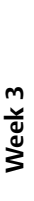 & 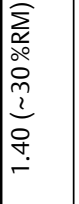 & 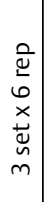 & 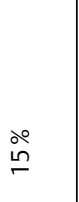 & \begin{tabular}{|l|}
$E$ \\
0 \\
$N$ \\
$\times$ \\
$o$ \\
$\grave{\omega}$ \\
$m$ \\
$m$
\end{tabular} & $\begin{array}{l}z \\
0 \\
u \\
\grave{z}\end{array}$ & 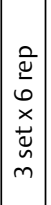 & 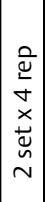 & $\Xi$ \\
\hline 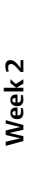 & 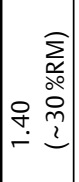 & 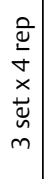 & $\begin{array}{l}\stackrel{\circ}{\circ} \\
\text { ஸn }\end{array}$ & 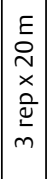 & $\mid \begin{array}{l}z \\
0 \\
\vdots \\
\Sigma \\
\Sigma\end{array}$ & 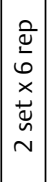 & 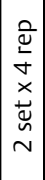 & 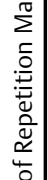 \\
\hline 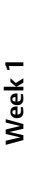 & 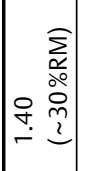 & 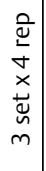 & 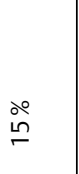 & 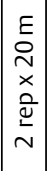 & $\begin{array}{l}z \\
0 \\
\cup \\
\vdots \\
z\end{array}$ & 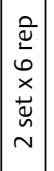 & & 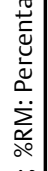 \\
\hline & 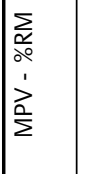 & $\begin{array}{l}\frac{\tilde{E}}{\mathrm{~g}} \\
\frac{\mathrm{g}}{\rho} \\
\end{array}$ & 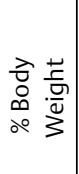 & 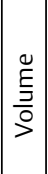 & 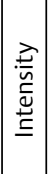 & 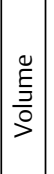 & $\frac{\mathscr{g}}{\mathrm{E}}$ & 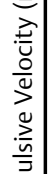 \\
\hline بू. & $\begin{array}{l}\frac{\pi}{0} \\
\overline{\bar{n}} \\
\overline{\overline{5}}\end{array}$ & & 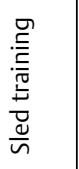 & & 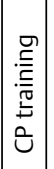 & & 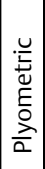 & 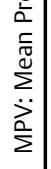 \\
\hline
\end{tabular}

\section{Lower limb power test}

Participants were placed into a front-step position, and they performed a one-step, horizontal acceleration by unwinding the tether until the end of the step (i. e., the CON phase), after which they returned to the initial position while rewinding the tether (i. e., the ECC phase). They also applied force throughout the course of the full CON phase at maximal velocity ( $\triangleright$ Fig. 1). The incremental test was performed on a conical pulley device (Costa Mesa, CA, USA; speed/force ratio level 1:3) and consisted of 3 sets of 6 maximal repetitions with different moments of inertia $\left(\mathrm{i}_{4}: 0.14, \mathrm{i}_{6}: 0.18\right.$, and $\mathrm{i}_{8}: 0.22 \mathrm{~kg} / \mathrm{m}^{2}$ ) for each leg. The three best CON power repetitions in the best set of the tests were selected for an analysis of the following variables: the mean power output in the CON phase (MPCON), the mean power output in the ECC phase (MPECC), and the ECC/CON ratio (ECC/CONrat). The power output was measured by using a rotatory encoder that recorded at a sampling frequency of $100 \mathrm{~Hz}$ (SmartCoach ${ }^{\mathrm{TM}}$, SmartCoach Europe AB, Stockholm, Sweden) and its associated software (SmartCoach ${ }^{\circledR}$ v.5.2.0.5). The reliabilities of the measures was assessed using the intraclass correlation coefficient (ICC) and the coefficient of variation (CV) and showed good reliability for all measures: ICC (0.80-0.81) [16] and CV (8.3-10.4\%) [17]. The smallest worthwhile changes for MPCON, MPECC, and ECC/CONrat were 3.4\% (CL: 2.5-5.5\%), $3.8 \%$ (2.8$6.3 \%)$, and $3.8 \%(2.8-6.3 \%)$, respectively.

\section{Statistical analysis}

Data are presented as the means \pm SDs. The reliability analysis was performed by using a two-way random ICC, with the absolute agreement, $90 \%$ confidence intervals, and the CV. Such an analysis was performed via the selected repetitions (i. e., the three best concentric MP) during the lower-limb tests. All of the data were log-transformed for the analysis, in order to reduce the bias arising from non-uniformity errors, after which the data were analysed for practical significance by using magnitude-based inferences [18]. The effect sizes (ES, $90 \%$ confidence limit [19]) of the selected variables were calculated by using the pooled pre-training SDs. The threshold values for the Cohen ES statistics were >0.2 (small), $>0.6$

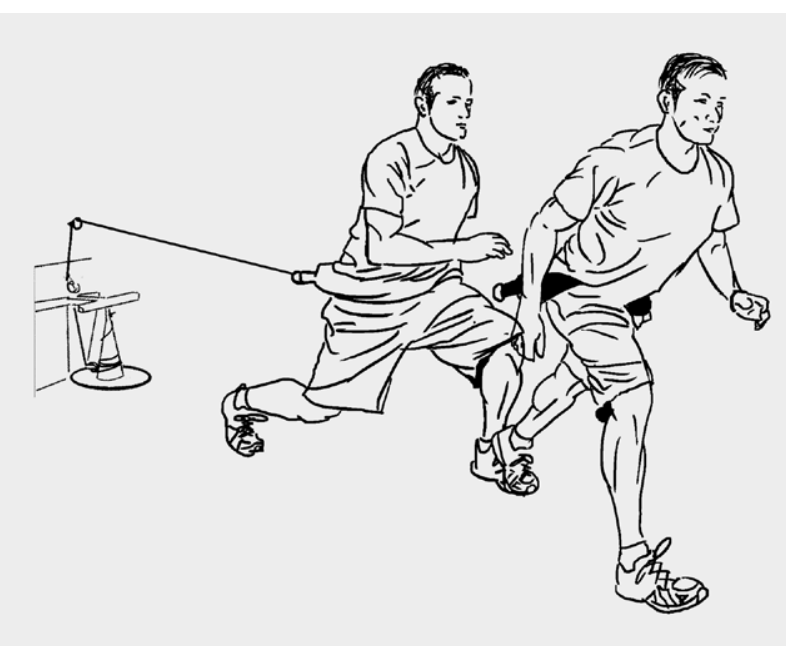

- Fig. 1 One-step horizontal acceleration in Conical Pulley device. 
(moderate), and $>1.2$ (large) [18]. The chance that any difference was better/greater (i. e., greater than the smallest worthwhile change, or SWC [ 0.2 multiplied by the between-subject standard deviation based on the Cohen's d principle, ES]), similar or worse/ smaller than that of the other group was subsequently calculated. The quantitative chances of beneficial/better or detrimental/poorer effects were qualitatively assessed as follows $[18,20]:<1 \%$, most likely not; $>1-5 \%$, very unlikely; $>5-25 \%$, unlikely; $>25-75$, possible; $>75-95 \%$, likely; $>95-99 \%$, very likely; and $>99 \%$, most likely. If there was a chance that the true value was $>25 \%$ beneficial and $>0.5 \%$ harmful, then the clinical effect was considered to be unclear [18]. However, the clinical inference was declared to be beneficial when the odds ratio of benefit/harm was $>66$ [18].

\section{Results}

The between-groups pre-test analysis showed no substantial differences in any of the variables with the lower limb power test. Nevertheless, the CG had a substantially better T10m (\%:- $2.9[90 \%$ CL: $-5.4 ;-0.8], E S=-0.83$ [90\%CL:-1.52;-0.14], 94\%/5\%/1\% with changes for lower/similar/greater values, respectively), $710-20 \mathrm{~m}$ (\%: - 4.3 [90\% CL: - 7.3; - 1.3], ES = - 1.02 [90\% CL:1.74; - 0.3], $97 \% / 3 \% / 1 \%$ with changes for lower/similar/greater values, respectively), and T20m (\%:- $3.6[90 \% \mathrm{CL}:-5.8 ;-1.3], \mathrm{ES}=-1.26[90 \% \mathrm{CL}$ : $2.07 ;-0.45], 79 \% / 0 \% / 21 \%$ with changes for lower/similar/greater values, respectively) than the CPG during the pre-test.

The relative changes and qualitative outcomes from the withingroups analyses are shown for the CPG and CG in $>$ Table 2. Substantial improvements were observed in MPCON and MPECC during the lower limb power test in both groups compared to those of the pre-test. Furthermore, the ECC/CONrat, the T10 and T20m were also substantially enhanced in the CPG, whereas the T10-20m and $\mathrm{T} 20 \mathrm{~m}$ were substantially enhanced in the CG.

The percentages of change between-groups are shown in ₹ Table 3. The improvements in the MPECC (\%: 51.5 [90\%CL: 3.2; 75.7]), and ECC/CONrat (\%: 38.4 [90\%CL: $14.9 ; 55.5]$ ), were substantially greater in the CPG than in the CG. Additionally, the MPCON (\%: 101.6 [ $90 \% \mathrm{CL}: 7 ; 279.7]$ ) was substantially greater in the CG than in the CPG.

\section{Discussion}

The aims of this study were to analyse the effects of inertial chronic strength training on the $\mathrm{CON}$ power, $\mathrm{ECC}$ power, $\mathrm{ECC} / \mathrm{CON}$ ratio, and $20 \mathrm{~m}$ linear sprint performance in elite young soccer players. The main findings of this study were: a) the improvements in the MPECC and ECC/CONrat were substantially greater in the CPG than in the CG; and b) the addition of a weekly, one-step horizontal acceleration exercise (with the use of a conical pulley device) to the usual strength training session, based on sled training, full squats, and plyometric exercise, improved accelerations when starting from a stopped position, but seemed to be insufficient into achieving greater improvements in the ability to sprint $20 \mathrm{~m}$ compared to the performance of typical strength training exercises alone.

Usually, a coach or physical trainer tends to associate the use of an inertial device with an increase in power output during the CON or ECC phases of movement used in training [12]. Based on the re-

\begin{tabular}{|c|c|c|c|c|c|c|c|c|}
\hline & 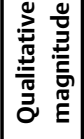 & 离 & 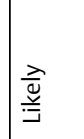 & $\mid$\begin{tabular}{|l}
$\frac{\vec{y}}{\bar{y}}$ \\
$\frac{\text { 产 }}{5}$
\end{tabular} & 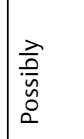 & $\mid \begin{array}{l}\stackrel{\vec{Q}}{\Xi} \\
\underline{\Xi}\end{array}$ & 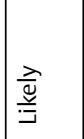 & $\begin{array}{l}\bar{u} \\
\underline{u}\end{array}$ \\
\hline & 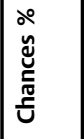 & $\frac{\frac{0}{\infty}}{\frac{\infty}{\sigma}}$ & 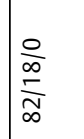 & $\underset{⿱ 亠}{\stackrel{N}{N}}$ & $\frac{\frac{\tilde{D}}{r}}{\frac{m}{r}}$ & $\begin{array}{l}\frac{8}{0} \\
\frac{2}{0}\end{array}$ & $\frac{\frac{\infty}{0}}{\frac{m}{a}}$ & 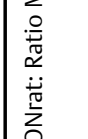 \\
\hline & $\frac{\overline{\tilde{u}}}{\tilde{u}}$ & 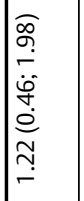 & 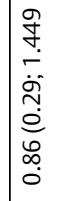 & 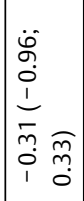 & 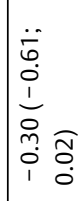 & 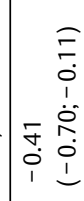 & 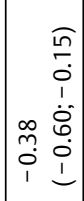 & 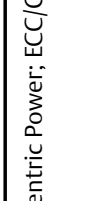 \\
\hline U & 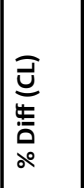 & 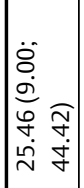 & 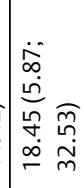 & 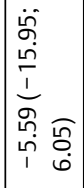 & 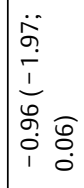 & 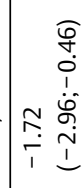 & 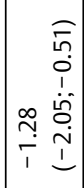 & 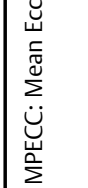 \\
\hline & 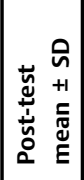 & 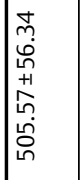 & 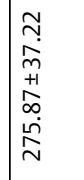 & $\mid \begin{array}{l}0 \\
0 \\
0 \\
+1 \\
0 \\
\hat{n} \\
0 \\
0\end{array}$ & 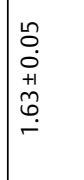 & 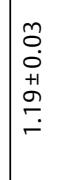 & 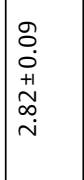 & 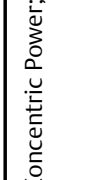 \\
\hline & 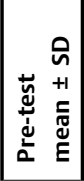 & 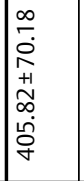 & $\begin{array}{l}\tilde{N} \\
\tilde{o} \\
\tilde{o} \\
+1 \\
\tilde{N} \\
\tilde{N} \\
\tilde{N} \\
\tilde{N}\end{array}$ & $\begin{array}{l}0 \\
0 \\
0 \\
+1 \\
0 \\
01 \\
0 \\
0\end{array}$ & 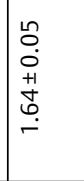 & 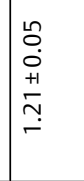 & 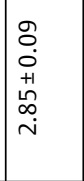 & 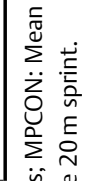 \\
\hline & 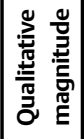 & 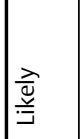 & 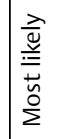 & $\mid \begin{array}{l}\frac{\overrightarrow{\underline{e}}}{\stackrel{\Xi}{\Xi}}\end{array}$ & 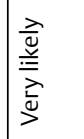 & 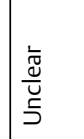 & 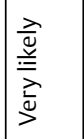 & 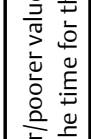 \\
\hline & 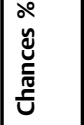 & $\frac{\frac{a}{\sqrt{n}}}{\frac{\Omega}{2}}$ & $\frac{0}{\frac{0}{2}}$ & $\frac{\frac{0}{0}}{\frac{0}{5}}$ & $\frac{\infty}{\frac{\infty}{d}}$ & $\frac{\frac{g}{g}}{\frac{g}{n}}$ & $\frac{\infty}{\frac{\infty}{v}}$ & t. \\
\hline & $\frac{\bar{z}}{y}$ & 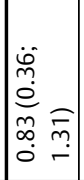 & 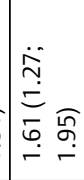 & 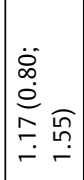 & 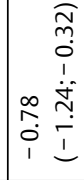 & 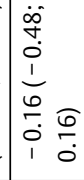 & 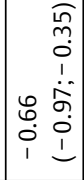 & 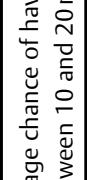 \\
\hline & 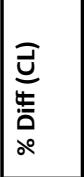 & 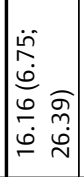 & 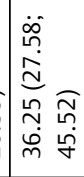 & 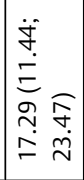 & 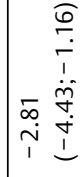 & 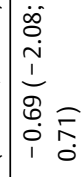 & 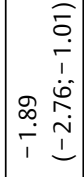 & 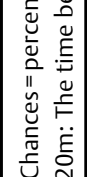 \\
\hline & 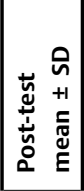 & 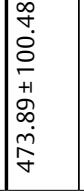 & 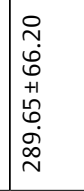 & \begin{tabular}{|l}
0 \\
0 \\
0 \\
+1 \\
0 \\
0 \\
0 \\
0
\end{tabular} & \begin{tabular}{|l}
0 \\
0 \\
0 \\
+1 \\
+1 \\
0 \\
0 \\
-1
\end{tabular} & 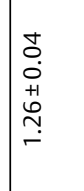 & 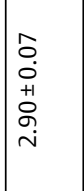 & 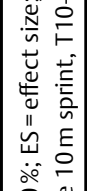 \\
\hline 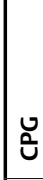 & 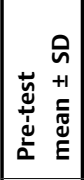 & 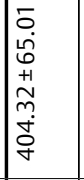 & 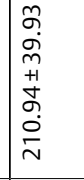 & $\begin{array}{l}0 \\
0 \\
0 \\
+1 \\
\\
\\
0\end{array}$ & $\begin{array}{l}0 \\
0 \\
0 \\
+1 \\
0 \\
0 \\
\dot{0} \\
\end{array}$ & 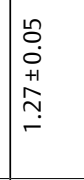 & $\begin{array}{l}\infty \\
0 \\
0 \\
+1 \\
0 \\
0 \\
i \\
i\end{array}$ & 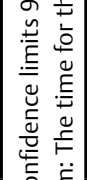 \\
\hline & \begin{tabular}{|l}
$\frac{y}{2}$ \\
$\frac{0}{0 \pi}$ \\
$\frac{\pi}{50}$ \\
$>$
\end{tabular} & 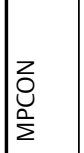 & \begin{tabular}{|l}
$\breve{u}$ \\
U. \\
$\Sigma$
\end{tabular} & 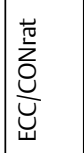 & 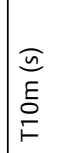 & 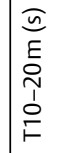 & $\begin{array}{l}\tilde{n} \\
E \\
\stackrel{N}{N} \\
F\end{array}$ & 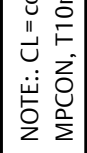 \\
\hline
\end{tabular}


- Table 3 Pre-post increased of CPG compared to CG.

\begin{tabular}{|l|l|l|l|l|l|l|}
\hline Variables & VPG mean \pm SD & CG mean \pm SD & \% Diff $(\mathrm{CL})$ & ES (CL) & $\begin{array}{l}\text { Chances \% } \\
\text { magnitude }\end{array}$ \\
\hline MPCON & $69.57 \pm 70.61$ & $99.75 \pm 107.12$ & $101.62(7.08 ; 279.65)$ & $0.71(0.07 ; 1.35)$ & $94 / 4 / 2$ & Likely \\
\hline MPECC & $78.71 \pm 36.96$ & $41.54 \pm 49.04$ & $-51.52(-75.71 ;-3.24)$ & $-1.38(-2.71 ;-0.06)$ & $3 / 5 / 92$ & Likely \\
\hline ECC/CONrat & $0.39 \pm 0.04$ & $0.27 \pm 0.11$ & $-38.47(-55.52 ;-14.91)$ & $-3.94(-6.57 ;-1.31)$ & $0 / 4 / 96$ & Very likely \\
\hline T10m (s) & $-0.05 \pm 0.05$ & $-0.02 \pm 0.03$ & $-45.90(-78.28 ; 34.78)$ & $-0.64(-1.59 ; 0.31)$ & $12 / 1 / 87$ & Unclear \\
\hline T10-20m (s) & $-0.01 \pm 0.03$ & $-0.02 \pm 0.03$ & $-4.8(-49.98 ; 119.28)$ & $0.05(-0.80 ; 0.90)$ & $54 / 2 / 45$ & Unclear \\
\hline T20m (s) & $-0.06 \pm 0.05$ & $-0.04 \pm 0.04$ & $-29.86(-55.86 ; 9.45)$ & $0.70(-1.58 ; 0.18)$ & $8 / 3 / 89$ & Unclear \\
\hline
\end{tabular}

NOTE:. $C L=$ confidence limits $90 \%$; ES = effect size; Chances = percentage chance of having better/similar/poorer values; MPCON: Mean Concentric

Power; MPECC: Mean Eccentric Power; ECC/CONrat: Ratio MPECC/MPCON, T10m: The time for the $10 \mathrm{~m}$ sprint, T10-20m: The time between 10 and

$20 \mathrm{~m}$ sprint, T20m: The time for the $20 \mathrm{~m}$ sprint.

sults of our study, the CPG obtained an increase in MPECC, which was substantially higher than that of the CG, whereas the CG substantially improved its MPCON more than the CPG. It is possible that the tendency of CG athletes to reproduce the patterns of movement of their usual training systems (i. e., sled training) when using a conical pulley device, allows them to produce a higher MPCON than the athletes of the CPG. Conversely, athletes who trained with the $\mathrm{CP}$ device tended to improve energy storage during the ECC phase, which allowed them to produce a higher MPECC than CG athletes. These results are in agreement with previous findings for the use of inertial devices, wherein the use of the conical pulley allowed for a greater mean eccentric force than the use of free-weight exercises, whereas the concentric force was higher with the use of free-weights than with the use of the conical pulley [21]. Notwithstanding, the MPCON and MPECC outcomes were improved in both groups (i. e., the CPG and CG); therefore, we cannot clearly state that this improvement is exclusively produced by training with the conical pulley. The ECC/CONrat was only substantially improved in the CPG. We could assume that the relationship between ECC and CON power outputs would be an outcome to consider when defining a functional strength training regimen with the use of a conical pulley.

The velocity of ECC contractions has a high correlation with sports performance, and the athlete's ability to sequence stiff muscles and compliant tendons for power production depends on the ECC utilization ratio $[22,23]$. Thus, it could be inferred that the increase in the mean ECC/CONrat reported in the present study would provide added training benefits in 10 and $20 \mathrm{~m}$ sprints. In our study, the CPG substantially improved the $\mathrm{T} 10 \mathrm{~m}(-2.8 \%, \mathrm{ES}=0.78)$. These improvements are very similar to those obtained by Gonzalo-Skok et al. [10], who utilized a biweekly training programme of 8 weeks of squat VP exercises $(-2.8 \%, E S=0.70)$ and multidirectional VP exercises $(-2.4 \%$, ES $=0.43)$. However, the T10m of our players $(1.69 \mathrm{~s})$ was significantly lower than that reported by Gonzalo-Skok et al. [10] (unidirectional, $1.82 \mathrm{~s}$; multidirectional, $1.81 \mathrm{~s}$ ) before starting the training, and only one of the six exercises carried out by the multidirectional training group is identical to that proposed in our study. De Hoyo et al. [13] analysed the effects of training (based on an exercise identical to the one proposed in this study) for a group of active subjects who trained for 6 weeks ( 3 times a week). They observed that the $\mathrm{T} 10 \mathrm{~m}(-0.01 \mathrm{~s})$ was significantly lower than that obtained in our study $(0.04 \mathrm{~s})$, although the athletes appeared to start from a simi- lar initial level. It is possible that the usual training routine for our CPG, based on the uses of full squat exercises, moderate sledding, and plyometric exercises, could have an additional effect of improving the ability to accelerate within $10 \mathrm{~m}$., although the CG exhibited no improvements in the $T 10 \mathrm{~m}$. These results are similar to those obtained by De Hoyo et al. [7], who used a protocol in which the different exercises included within our training routine were compared separately. Nevertheless, our results do not correspond to those obtained by Otero-Esquina et al. [8], wherein participants performed a routine similar to the one proposed in this study, but with the addition of an exercise (YoYo ${ }^{\text {TM }}$ leg curl) with special emphasis on the ECC phase of the hamstring muscle, thus allowing the $\operatorname{T10m}(-1.4 \%$, $\mathrm{ES}=0.43$ ) to improve but only in the group that trained two times a week. It is possible that the application of this device twice a week in the ECC phase of the hamstring musculature could be an important factor in the improvement of the acceleration capacity in the $10 \mathrm{~m}$ sprint, although this effects has currently only been demonstrated for distances of $20 \mathrm{~m}$ [24] and $30 \mathrm{~m}$ [9]. Our study did not demonstrate substantial differences between the groups in $710 \mathrm{~m}$, but the results exhibited a noticeably greater improvement than the results from another study with young soccer players [8]. It is possible that the lower capacity to accelerate from the CPG to the CG in the pretest could explain these differences, which indicate a greater capacity for improvement in the CPG; therefore, further research concerning the influence of the conical pulley ECC/CONrat on the sprint performance is needed.

The CPG did not exhibited improvements in the $T 10-20 \mathrm{~m}$. It is known that a conical pulley strength training regimen improves ground reaction forces at high velocities while moving in multiple planes [25]. To be effective with this device, the subject must apply force throughout the course of the CON phase of the movement, thus producing force for a longer time period than in other systems [21]. It is possible that this greater time period of force application would not provide additional training benefits when the time of the application of the force tends to be minimal (e. g., T10-20 m). The CG had an improvement in T10-20 m ( $-1.72 \%$, ES $=0.41)$. These results are very similar to those obtained by Otero-Esquina et al. [8] ( $-1.8 \%$, ES = 0.52) with the use of similar protocols; however, De Hoyo et al. [7] isolated each of the proposed exercises, and showed a substantial improvement only for the group that trained with full squats $(-1.9 \%, E S=0.61)$. In our study, both groups exhibited improvements in $\mathrm{T} 20 \mathrm{~m}$, without substantial between- 
groups differences. However, de Hoyo et al. [7] did not demonstrate an improvement in the $\mathrm{T} 20 \mathrm{~m}$ in any of the exercises proposed in our typical training routine, and Otero-Esquina et al. [8] showed a reduction $(-1.5 \mathrm{~s}, \mathrm{ES}=0.47)$ that was significantly lower than that of the CPG; therefore further research concerning the influence of the CP ECC/CONrat on the force application sequence is needed.

This study had a limitation that should be acknowledged. The CPG performed an exercise to a greater degree than the CG during the training routines. It is possible that more work could explain the greater amount of results or higher levels of fatigue. However, this study analysed the effects of the addition an exercise that had a unique impact on the ECC phase of the movement, compared to its usual strength training session based on sled training, full squats, and plyometrics. This usual strength training session, combined with an exercise that had a unique impact on the ECC phase of the movement, showed improvements in the sprints of young soccer players [8], but no analyses were performed on the isolated effects of addition of the ECC exercise. Therefore, the current design was needed, in order to analyse the isolated effect of the addition of the ECC exercise.

This study provides very important practical implications for those physical trainers who intend to use a rotating inertial device, such as the conical pulley device. The use of conical pulley horizontal acceleration exercises optimizes the mean power outputs in the CON and ECC phases, but mainly optimizes the relationship between the ECC/CON. The application of this device once a week does not appear to be enough to fully benefit from this device; however, the producing force for a longer time period than in other systems [20], could be an important factor in the improvement of the short acceleration capacity (e.g., T10m), and would not provide additional training benefits when the time of the application of the force tends to be minimal (e. g., T10-20 m).

\section{Conclusions}

To be efficient when defining inertial chronic strength training, the mean power outputs need to be measured during the CON and ECC phases and an analysis of the relationship between the ECC and CON power outputs should be included. Adding a weekly, one-stepacceleration exercise with a conical pulley device provides insufficient data for an improvement in the ability to sprint in $10 \mathrm{~m}$ and $20 \mathrm{~m}$ with the used of the conical pulley device, compared to strength training with the use of sled training, full squats, and plyometric exercises.

\section{Acknowledgements}

We would like to acknowledgement Dr. Hernandez Abad the design of the Figure for this study.

\section{Conflict of Interest}

Authors declare that they have no conflict of interest.

\section{References}

[1] Morin JB, Petrakos G, Jimenez-Reyes P, Brown SR, Samozino P, Cross MR. Very-heavy sled training for improving horizontal-force output in soccer players. Int J Sports Physiol Perform 2017; 12: 840-844

[2] Morin JB, Samozino P. Interpreting power-force-velocity profiles for individualized and specific training. Int J Sports Physiol Perform 2016; 11: 267-272

[3] Norrbrand L, Fluckey JD, Pozzo M, Tesch PA. Resistance training using eccentric overload induces early adaptations in skeletal muscle size. Eur J Appl Physiol 2008; 102: 271-281

[4] Norrbrand L, Pozzo M, Tesch PA. Flywheel resistance training calls for greater eccentric muscle activation than weight training. Eur J Appl Physiol 2010; 110: 997-1005

[5] Javier Nunez F, Suarez-Arrones L], Cater P, Mendez-Villanueva A. The High-Pull Exercise: A comparison between a versapulley flywheel device and the free weight. Int J Sports Physiol and Perform 2017; 12: 527-532

[6] Maroto-Izquierdo S, Garcia-Lopez D, Fernandez-Gonzalo R, Moreira OC, Gonzalez-Gallego J, de Paz JA. Skeletal muscle functional and structural adaptations after eccentric overload flywheel resistance training: a systematic review and meta-analysis. J Sci Med Sport 2017; 20: 943-951

[7] de Hoyo M, Gonzalo-Skok O, Sanudo B, Carrascal C, Plaza-Armas JR, Camacho-Candil F et al. Comparative effects of in-season full-back squat, resisted sprint training, and plyometric training on explosive performance in U-19 elite soccer players. J Strength Cond Res 2016; 30: $368-377$

[8] Otero-Esquina C, de Hoyo Lora M, Gonzalo-Skok O, Dominguez-Cobo S, Sanchez $\mathrm{H}$. Is strength-training frequency a key factor to develop performance adaptations in young elite soccer players? Eur J Sport Sci 2017; 17: 1241-1251

[9] Askling C, Karlsson J, Thorstensson A. Hamstring injury occurrence in elite soccer players after preseason strength training with eccentric overload. Scand J Med Sci Sports 2003; 13: 244-250

[10] Gonzalo-Skok O, Tous-Fajardo ], Valero-Campo C, Berzosa C, Bataller $\mathrm{AV}$, Arjol-Serrano JL et al. Eccentric-overload training in team-sport functional performance: constant bilateral vertical versus variable unilateral multidirectional movements. Int J Sports Physiol Perform 2017; 12: 951-958

[11] Tous-Fajardo J, Gonzalo-Skok O, Arjol-Serrano JL, Tesch P. Enhancing change-of-direction speed in soccer players by functional inertial eccentric overload and vibration training. Int I Sports Physiol Perform 2016; 11: 66-73

[12] Carmona G, Guerrero M, Cusso R, Padulles JM, Moras G, Lloret M et al. Muscle enzyme and fiber type-specific sarcomere protein increases in serum after inertial concentric-eccentric exercise. Scand J Med Sci Sports 2015; 25: e547-e557

[13] de Hoyo M, Sanudo B, Carrasco L, Dominguez-Cobo S, Mateo-Cortes ], Cadenas-Sanchez MM et al. Effects of traditional versus horizontal inertial flywheel power training on common sport-related tasks. J Hum Kinet 2015; 47: 155-167

[14] World Medical Association Declaration of Helsinki. Ethical principles for medical research involving human subjects. JAMA. 2013; 310: 2191-2194

[15] Harriss DJ, Macsween A, Atkinson G. Standards for ethics in sport and exercise science research: 2018 update. Int J Sports Med 2017; 38: 14: 1126-1131

[16] Koo TK, Li MY. A Guideline of selecting and reporting intraclass correlation coefficients for reliability research. J Chiropr Med 2016; 15: 155-163

[17] Cormack SJ, Newton RU, McGuigan MR, Doyle TL. Reliability of measures obtained during single and repeated countermovement jumps. Int J Sports Physiol Perform 2008; 3: 131-144 
[18] Hopkins WG, Marshall SW, Batterham AM, Hanin J. Progressive statistics for studies in sports medicine and exercise science. Med Sci Sports Exerc 2009; 41: 3-13

[19] Garcia-Hermoso A, Cavero-Redondo I, Ramirez-Velez R, Ruiz JR, Ortega $\mathrm{FB}$, Lee DC et al. Muscular strength as a predictor of all-cause mortality in an apparently healthy population: a systematic review and meta-analysis of data from approximately 2 million men and women. Arch Phys Med Rehabil 2018; 99: 2100-2113

[20] Batterham AM, Hopkins WG. Making meaningful inferences about magnitudes. Int J Sports Physiol Perform 2006; 1: 50-57

[21] Nunez F], Suarez-Arrones LJ, Cater P, Mendez-Villanueva A. The High-pull exercise: A comparison between a versapulley flywheel device and the free weight. Int J Sports Physiol Perform 2017; 12: 527-532
[22] Katz B. The relation between force and speed in muscular contraction. J Physiol 1939; 96: 45-64

[23] Komi PV, Buskirk ER. Effect of eccentric and concentric muscle conditioning on tension and electrical activity of human muscle. Ergonomics 1972; 15: 417-434

[24] de Hoyo M, Pozzo M, Sanudo B, Carrasco L, Gonzalo-Skok O, Dominguez-Cobo $S$ et al. Effects of a 10 -week in-season eccentricoverload training program on muscle-injury prevention and performance in junior elite soccer players. Int J Sports Physiol Perform 2015; 10: $46-52$

[25] Moras G, Vazquez-Guerrero ]. Force production during squats performed with a rotational resistance device under stable versus unstable conditions. J Phys Ther Sci 2015; 27: 3401-3406 